\title{
Twisted modules and pseudo-endomorphisms
}

\author{
Haisheng Li \\ Department of Mathematical Sciences \\ Rutgers University, Camden, NJ 08102
}

\begin{abstract}
We exhibit a connection between two constructions of twisted modules for a general vertex operator algebra with respect to inner automorphisms. We also study pseudo-derivations, pseudo-endomorphisms, and twist deformations of ordinary modules by pseudo-endomorphisms, which are intrinsically connected to one of the two constructions.
\end{abstract}

\section{Introduction}

In vertex operator algebra theory, for a vertex operator algebra $V$, in addition to the notion of $V$-module there is a notion of $\sigma$-twisted $V$-module where $\sigma$ is a finite order automorphism of $V$. This is one of those new features of vertex operator algebras, in contrast with classical Lie or associative algebras. The notion of twisted module was originated from the construction of the celebrated moonshine module vertex operator algebra $V^{\natural}$ (see [FLM1, [FLM2, [Le]) and it had played a key role therein. Since then, the theory of twisted representations has been extensively studied in literature (cf. [FFR], [D], [Li1], [DLM3]).

In [Li1], a conceptual construction of vertex algebras and their twisted modules was obtained and a canonical construction of twisted modules with respect to inner automorphisms was found. Let $V$ be a general vertex operator algebra and let $h \in V$, satisfying that $L(n) h=\delta_{n, 0} h$ and $h(n) h=\delta_{n, 1} \alpha$ for $n \in \mathbb{N}$, where $\alpha$ is a rational number. Assume that $h(0)$ acts semisimply on $V$ with eigenvalues in $\frac{1}{r} \mathbb{Z}$ for some positive integer $r$. Note that $e^{2 \pi i h(0)}$, denoted by $\sigma_{h}$, is an automorphism of $V$. Set

$$
\Delta(h, z)=z^{h(0)} \exp \left(\sum_{n \geq 1} \frac{h(n)}{-n} z^{-n}\right) .
$$

It was proved therein that for any $V$-module $\left(W, Y_{W}(\cdot, z)\right),\left(W, Y_{W}(\Delta(h, z) \cdot, z)\right)$ carries the structure of a $\sigma_{h}$-twisted $V$-module. This correspondence gives rise to a canonical isomorphism between the category of $V$-modules and the category of $\sigma_{h}$-twisted $V$-modules.

There is another construction of twisted modules by employing the construction of contragredient modules. Let $V$ be a vertex operator algebra. A fundamental result due to FHL is that for any $V$-module $W$, the restricted dual $W^{\prime}$ of $W$ has a natural $V$-module structure, called the contragredient module. Note that the construction of contragredient module depends on the conformal vector; it depends not only on the vertex algebra structure. In [DLM2, to study modules for vertex operator 
algebras associated to affine Lie algebra $\hat{s l}_{2}$ at admissible levels, we employed a different conformal vector whose $L(0)$-weight grading corresponds to the principal grading, whereas the usual Segal-Sugawara conformal vector corresponds to the homogeneous grading. More generally, let $V$ be a vertex operator algebra with conformal vector $\omega$ and let $h \in V$ be such that $L(n) h=\delta_{n, 0} h$ and $h(n) h=\delta_{n, 1} \alpha$ for $n \in \mathbb{N}$, where $\alpha$ is a rational number. Then $\omega+L(-1) h$ is a conformal vector. (This is a reformulation of the Feigin-Fuchs construction of modules for the Virasoro algebra.) In general, vertex algebra $V$ equipped with the new conformal vector $\omega+L(-1) h$ is a $\mathbb{Q}$-graded vertex operator algebra, whose notion differs from the ordinary one only on the grading group. It was observed therein that for a general $\mathbb{Q}$-graded vertex operator algebra $V$, essentially the same argument of [FHL] shown that the contragredient dual of a $V$-module is a twisted module with respect to the automorphism $\sigma=e^{4 \pi i L(0)}$. Then, given an ordinary vertex operator algebra $V$ with an element $h \in V$ satisfying the conditions as above, we have a different construction of twisted modules by considering contragredient dual of $V$-modules with respect to the new conformal vector associated to $h$.

This present paper is to study the relation between the two constructions of twisted modules and to study a certain general theory closely related to the first construction. More specifically, in the first part, we give a connection between the two constructions of twisted modules and in the second we study pseudo-endomorphisms and the twist deformations of ordinary modules by pseudo-endomorphisms.

Note that the first construction is simply to twist ordinary representations. Closely related to this spirit is the physics super-selection theory (cf. [HK], [FRS]). For an illustration, let $A$ be an associative $\left(C^{*}\right.$-)algebra. A trivial fact is that for any endomorphism $\sigma$ of $A$ and for any representation $\rho$ of $A, \rho \circ \sigma$ is also a representation of $A$. In general, $A$ has infinitely many nonequivalent irreducible modules, but in practice only certain irreducible modules are selected by certain rules. The super selection rule is to select those representations obtained from the distinguished "vacuum module" through (unitary) endomorphisms. Also in this very theory, fusion rule can be defined and studied in terms of endomorphisms. For vertex operator algebras, the adjoint modules are understood to be the vacuum modules. All of these motivated the work [Li2] in which the physics super-selection theory was studied in the contexts of vertex operator algebras. The key idea is to replace endomorphisms with "pseudo-endomorphisms" like $\Delta(h, z)$, which are not endomorphisms in the usual sense. (Notice that if $V$ is a simple vertex operator algebra, the $\sigma$-twist of $V$ is always isomorphic to $V$ for any endomorphism $\sigma$ of $V$.) The "pseudoendomorphisms" considered in [Li2] are elements $\Delta(z) \in \operatorname{Hom}\left(V, V \otimes \mathbb{C}\left[z, z^{-1}\right]\right)$, satisfying

$$
\begin{aligned}
& \Delta(z) \mathbf{1}=\mathbf{1}, \quad[L(-1), \Delta(z)]=-\frac{d}{d z} \Delta(z), \\
& \Delta(z) Y\left(v, z_{0}\right)=Y\left(\Delta\left(z+z_{0}\right) v, z_{0}\right) \Delta(z)
\end{aligned}
$$

for $v \in V$. It was proved therein that for any $V$-module $\left(W, Y_{W}(\cdot, z)\right)$, the pair 
$\left(W, Y_{W}(\Delta(z) \cdot, z)\right)$ also carries the structure of a $V$-module. Furthermore, fusion rule in the sense of [FHL] was studied in terms of "pseudo-endomorphisms." In particular, it was proved that twisting the adjoint module $V$ by $\Delta(h, z)$ gives simple currents in a certain sense.

The notion of pseudo-endomorphism was formally introduced and further studied later in [Li4]. Motivated by Etingof-Kazhdan's notion of pseudo-derivation (see [EK]), we defined a pseudo-endomorphism of $V$ more generally as an element $\Delta(z) \in$ $\operatorname{Hom}(V, V \otimes \mathbb{C}((z)))$ with the same set of axioms, whereas a pseudo-derivation of $V$ is an element $\psi(z) \in \operatorname{Hom}(V, V \otimes \mathbb{C}((z)))$ such that

$$
[L(-1), \psi(z)]=-\frac{d}{d z} \psi(z), \quad[\psi(z), Y(v, x)]=Y(\psi(z+x) v, x)
$$

for $v \in V$. Just as with the classical case, the exponential of a pseudo-derivation, provided it exists, is a pseudo-automorphism. A result proved in [EK] is that for any $f(z) \in \mathbb{C}((z)), v \in V$,

$$
X_{v, f}(z):=\sum_{n \geq 0} \frac{f^{(n)}(z)}{n !} v_{n}
$$

is a pseudo-derivation of $V$. As it was observed in [Li4] (Remark 3.9), if we could take $f(z)=\log z$, we would have

$$
X_{h, f}=h_{0} \log z+\sum_{n \geq 1}(-1)^{n-1} \frac{1}{n} h_{n} z^{-n}
$$

and $\Delta(h, z)=e^{X_{h, f}(z)}$. As the exponentials of pseudo-derivations "are" pseudoautomorphisms, the importance of exponentiating objects like $X_{h, f}$ is manifest. This suggests to study "logarithmic" pseudo-derivations and pseudo-endomorphisms, which are elements of $\operatorname{Hom}(V, V \otimes \mathbb{C}((z))[\log z])$.

The second part of this present paper is a continuation of [Li4], to study pseudoderivations, their formal exponentials, and pseudo-endomorphisms. More specifically, we study more general pseudo-derivations and pseudo-endomorphisms with $\mathbb{C}((z))$ replaced by certain commutative algebras $R$ over $\mathbb{C}((z))$ such as $\mathbb{C}((z))[\log z]$ and $\mathbb{C}\left(\left(z^{1 / r}\right)\right)$ with $r$ a positive integer. We also study pseudo-endomorphism twists of ordinary representations for vertex algebras. For the vertex operator algebra associated to a Heisenberg algebra, we show that certain pseudo-endomorphism twists of the adjoint module gives non-highest weight modules.

Recently, Huang (see $[\mathrm{H}]$ ) obtained a very interesting generalization of the first construction, where $h(0)$ is allowed to be locally finite, instead of semisimple. In his generalization, the factor $z^{h(0)}$ in the construction is essentially replaced with $e^{h(0) \log z}$ in a certain natural way. Then Huang obtained what he called generalized twisted modules with respect to an automorphism which is no longer of finite order.

This paper is organized as follows: In Section 2, we give a connection between the two constructions of twisted modules. In Section 3, we study more general pseudo-derivations and pseudo-endomorphisms. 


\section{Two constructions of $\sigma_{h}$-twisted modules}

In this section, we first review the two constructions of twisted modules for an inner automorphism and we then give a connection between the two constructions.

We start with some basic notions. An automorphism of a vertex algebra $V$ is a bijective linear endomorphism $\sigma$ of $V$ such that $\sigma(\mathbf{1})=\mathbf{1}$ and $\sigma(Y(u, z) v)=$ $Y(\sigma(u), z) \sigma(v)$ for $u, v \in V$. An automorphism of a vertex operator algebra $V$ is an automorphism $\sigma$ of $V$ viewed as a vertex algebra such that $\sigma(\omega)=\omega$, where $\omega$ is the conformal vector of $V$. Consequently, every automorphism of a vertex operator algebra preserves all the homogeneous ( $L(0)$-weight) subspaces.

A simple fact is that for any vertex algebra $V$ and for any $a \in V, e^{a_{0}}$ is an automorphism of $V$, provided that $a_{0}$ is locally finite on $V$. Assume that $V$ is a vertex operator algebra. Let $a \in V_{(1)}$, i.e., $a \in V$ with $L(0) a=a$. It follows that $a_{0}$ preserves the $L(0)$-weight spaces of $V$, which implies that $a_{0}$ is locally finite on $V$. Thus $e^{a_{0}}$ is an automorphism of $V$ viewed as a vertex algebra. Furthermore, if $a$ is primary in the sense that $L(n) a=0$ for $n \geq 1$, we have $e^{a_{0}}(\omega)=\omega$, so that $e^{a_{0}}$ is an automorphism of vertex operator algebra $V$.

Let $V$ be a vertex algebra and let $\sigma$ be an automorphism of $V$ of order $k$. A $\sigma$-twisted $V$-module (see [FLM2], [FFR], [D]) is a vector space $W$, equipped with a linear map

$$
\begin{aligned}
Y_{W}: \quad V & \rightarrow \operatorname{Hom}\left(W, W\left(\left(x^{1 / k}\right)\right)\right) \subset(\operatorname{End} W)\left[\left[x^{1 / k}, x^{-1 / k}\right]\right] \\
v & \mapsto Y_{W}(v, x)
\end{aligned}
$$

satisfying the conditions that $Y_{W}(\mathbf{1}, x)=1$,

$$
Y_{W}(\sigma v, x)=\lim _{x^{1 / k} \rightarrow \omega_{k}^{-1} x^{1 / k}} Y_{W}(v, x)
$$

for $v \in V$, where $\omega_{k}=\exp (2 \pi i / k)$, and that for $u \in V^{j}, v \in V$ with $0 \leq j \leq k-1$,

$$
\begin{gathered}
x_{0}^{-1} \delta\left(\frac{x_{1}-x_{2}}{x_{0}}\right) Y_{W}\left(u, x_{1}\right) Y_{W}\left(v, x_{2}\right)-x_{0}^{-1} \delta\left(\frac{x_{2}-x_{1}}{-x_{0}}\right) Y_{W}\left(v, x_{2}\right) Y_{W}\left(u, x_{1}\right) \\
=x_{2}^{-1}\left(\frac{x_{1}-x_{0}}{x_{2}}\right)^{-\frac{j}{k}} \delta\left(\frac{x_{1}-x_{0}}{x_{2}}\right) Y_{W}\left(Y\left(u, x_{0}\right) v, x_{2}\right)
\end{gathered}
$$

( $\sigma$-twisted Jacobi identity), where

$$
V^{j}=\left\{a \in V \mid \sigma(a)=\omega_{k}^{j} a\right\}
$$

If $V$ is a vertex operator algebra, a $\sigma$-twisted $V$-module, which by definition is $\mathbb{C}$-graded by the $L(0)$-weight, satisfies the two grading restrictions.

Let $V$ be a vertex operator algebra and let $h \in V$ be such that

$$
L(n) h=\delta_{n, 0} h, \quad h_{n} h=\delta_{n, 1} \gamma \mathbf{1} \text { for } n \in \mathbb{N},
$$


where $\gamma$ is a fixed rational number, and such that $h_{0}$ acts semisimply on $V$ with rational eigenvalues. In view of Borcherds' commutator formula we have

$$
\left[L(m), h_{n}\right]=-n h_{m+n}, \quad\left[h_{m}, h_{n}\right]=m \gamma \delta_{m+n, 0}
$$

for $m, n \in \mathbb{Z}$. We shall also freely use $h(n)$ for $h_{n}$.

Set

$$
\Delta(h, z)=z^{h(0)} \exp \left(\sum_{k=1}^{\infty} \frac{h(k)}{-k}(-z)^{-k}\right) \in(\operatorname{End} V)\{z\} .
$$

Note that $e^{2 \pi i h(0)}$ is an automorphism of $V$. Set $\sigma_{h}=e^{2 \pi i h(0)}$ and assume that $\sigma_{h}$ is of finite order. The following proposition was proved in [Li1]:

Proposition 2.1. Let $V$ be a vertex operator algebra and let $h \in V$ be such that (2.2) holds and such that $h(0)$ acts semisimply on $V$ with rational eigenvalues. Let $\left(W, Y_{W}(\cdot, z)\right)$ be a $V$-module. Then $\left(W, Y_{W}(\Delta(h, z) \cdot, z)\right)$ carries the structure of a $\sigma_{h}$-twisted $V$-module.

The following is essentially the Feigin-Fuchs bosonization of Virasoro algebra (cf. [DLinM]):

Proposition 2.2. Let $V$ be a vertex operator algebra with conformal vector $\omega$ of central charge $c$ and $h \in V$, satisfying (2.2). Then $\omega+\frac{1}{2} L(-1) h$ is a conformal vector of central charge $c-3 \gamma$.

In Proposition 2.2, $V$ with the new conformal vector $\tilde{\omega}$ in general is a $\mathbb{Q}$-graded vertex operator algebra in the following sense (see [DLM2]):

Definition 2.3. If $V$ satisfies all the axioms of a vertex operator algebra except that $V$ is $\mathbb{Q}$-graded instead of $\mathbb{Z}$-graded, we call $V$ a $\mathbb{Q}$-graded vertex operator algebra.

Let $V$ be a general $\mathbb{Q}$-graded vertex operator algebra. For $u \in V_{(\alpha)}$ with $\alpha \in \mathbb{Q}$ and for $n \in \mathbb{Z}$, we have (see [FHL])

$$
\left[L(0), u_{n}\right]=(\alpha-n-1) u_{n} .
$$

For $u \in V_{(\alpha)}, v \in V_{(\beta)}$ with $\alpha, \beta \in \mathbb{Q}$, we have

$$
e^{2 \pi i L(0)}\left(u_{n} v\right)=e^{2(\alpha+\beta-n-1) \pi i}\left(u_{n} v\right)=e^{2(\alpha+\beta) \pi i}\left(u_{n} v\right)=\left(e^{2 \pi i L(0)} u\right)_{n}\left(e^{2 \pi i L(0)} v\right) .
$$

Since $L(0) \mathbf{1}=\omega_{1} \mathbf{1}=0$ and $L(0) \omega=2 \omega$, we have

$$
e^{2 \pi i L(0)}(\mathbf{1})=\mathbf{1}, \quad e^{2 \pi i L(0)}(\omega)=\omega .
$$

Thus $\tau:=e^{2 \pi i L(0)}$ is an automorphism of $V$. It is clear that $\tau$ is of finite order if and only if there is a positive integer $T$ such that all the $L(0)$-weights, i.e., the $L(0)$ eigenvalues, are contained in $\frac{1}{T} \mathbb{Z}$, which is true if $V$ as a vertex algebra is finitely generated. 
Now, we assume that $V=\oplus_{\alpha \in \frac{1}{2 r} \mathbb{Z}} V_{(\alpha)}$ for some positive integer $r$. Then

$$
\tau^{2 r}=e^{4 r \pi i L(0)}=1 \text { on } V
$$

That is, $2 r$ is a period of $\tau$ and $r$ is a period of $\tau^{2}$.

Let $W=\oplus_{\alpha \in \mathbb{C}} W_{(\alpha)}$ be a $V$-module. Following [FHL], set

$$
W^{\prime}=\oplus_{\alpha \in \mathbb{C}} W_{(\alpha)}^{*}
$$

(the restricted dual of $W$ ) and define

$$
\left\langle Y_{W^{\prime}}(v, z) w^{\prime}, w\right\rangle=\left\langle w^{\prime}, Y_{W}\left(e^{z L(1)} e^{\pi i L(0)} z^{-2 L(0)} v, z^{-1}\right) w\right\rangle
$$

for $v \in V, w^{\prime} \in W^{\prime}, w \in W$. From the argument in [FHL], we also have

$$
\left\langle w^{\prime}, Y(v, z) w\right\rangle=\left\langle Y^{\prime}\left(e^{z L(1)} e^{-\pi i L(0)} z^{-2 L(0)} v, z^{-1}\right) w^{\prime}, w\right\rangle .
$$

The following was observed in DLM2]:

Proposition 2.4. Let $V$ be a $\mathbb{Q}$-graded vertex operator algebra such that $V=$ $\oplus_{\alpha \in \frac{1}{2 r} \mathbb{Z}} V_{(\alpha)}$ for some positive integer $r$ and let $W$ be a $V$-module. Then $\left(W^{\prime}, Y_{W^{\prime}}\right)$ carries the structure of a $\sigma$-twisted $V$-module with $\sigma=e^{4 \pi i L(0)}\left(=\tau^{2}\right)$.

Proof. It is just a slight modification of the proof of the contragredient module theorem in [FHL]. First, as the $L(0)$-eigenvalues are contained in $\frac{1}{2 r} \mathbb{Z}$, we see from (2.6) that for $v \in V$,

$$
Y_{W^{\prime}}(v, z) \in(\operatorname{End} W)\left[\left[z^{1 / r}, z^{-1 / r}\right]\right]
$$

and we have

$$
Y_{W^{\prime}}(v, z) \in \operatorname{Hom}\left(W, W\left(\left(z^{1 / r}\right)\right)\right)
$$

from the grading restrictions on $W$ as in FHL. Second, for $v \in V$ we have

$$
\lim _{z^{1 / r} \rightarrow \omega_{r}^{-1} z^{1 / r}} z^{-2 L(0)} v=\lim _{z^{1 / r} \rightarrow \omega_{r}^{-1} z^{1 / r}}\left(z^{1 / r}\right)^{-2 r L(0)} v=e^{4 \pi i L(0)} z^{-2 L(0)} v
$$

so that

$$
Y_{W^{\prime}}(\sigma v, z)=Y_{W^{\prime}}\left(e^{4 \pi i L(0)} v, z\right)=\lim _{z^{1 / r} \rightarrow \omega_{r}^{-1} z^{1 / r}} Y_{W^{\prime}}(v, z)
$$

where $\omega_{r}=\exp (2 \pi i / r) \in \mathbb{C}$. Then it remains to prove the twisted Jacobi identity. Let $a \in V_{(\alpha)}, b \in V$ with $\alpha \in \frac{1}{2 r} \mathbb{Z}$. We need to prove

$$
\begin{aligned}
& z_{0}^{-1} \delta\left(\frac{z_{1}-z_{2}}{z_{0}}\right) Y_{W^{\prime}}\left(a, z_{1}\right) Y_{W^{\prime}}\left(b, z_{2}\right)-z_{0}^{-1} \delta\left(\frac{z_{2}-z_{1}}{-z_{0}}\right) Y_{W^{\prime}}\left(b, z_{2}\right) Y_{W^{\prime}}\left(a, z_{1}\right) \\
& =z_{2}^{-1}\left(\frac{z_{1}-z_{0}}{z_{2}}\right)^{-2 \alpha} \delta\left(\frac{z_{1}-z_{0}}{z_{2}}\right) Y_{W^{\prime}}\left(Y\left(a, z_{0}\right) b, z_{2}\right)
\end{aligned}
$$


Recall the following formulas

$$
\begin{aligned}
z^{L(0)} L(-1) & =z^{-1} L(-1) z^{L(0)} \\
e^{z L(1)} L(-1) & =L(-1) e^{z L(1)}+2 z e^{z L(1)} L(0)-z^{2} e^{z L(1)} L(1), \\
z^{L(0)} Y\left(a, z_{0}\right) z^{-L(0)} & =Y\left(z^{L(0)} a, z_{0} z\right), \\
e^{z L(1)} Y\left(a, z_{0}\right) e^{-z L(1)} & =Y\left(e^{z\left(1-z z_{0}\right)}\left(1-z_{0} z\right)^{-2 L(0)} a, \frac{z_{0}}{1-z z_{0}}\right) .
\end{aligned}
$$

For $u, v \in V$, from [FHL we have

$$
\begin{aligned}
& z_{0}^{-1} \delta\left(\frac{z_{1}-z_{2}}{z_{0}}\right) Y_{W}\left(v, z_{2}^{-1}\right) Y_{W}\left(u, z_{1}^{-1}\right) \\
& \quad-z_{0}^{-1} \delta\left(\frac{z_{2}-z_{1}}{-z_{0}}\right) Y_{W}\left(u, z_{1}^{-1}\right) Y_{W}\left(v, z_{2}^{-1}\right) \\
= & -\left(z_{1} z_{2}\right)^{-1}\left(-\frac{z_{0}}{z_{1} z_{2}}\right)^{-1} \delta\left(\frac{-z_{2}^{-1}+z_{1}^{-1}}{-\frac{z_{0}}{z_{1} z_{2}}}\right) Y_{W}\left(v, z_{2}^{-1}\right) Y_{W}\left(u, z_{1}^{-1}\right) \\
& +\left(z_{1} z_{2}\right)^{-1}\left(-\frac{z_{0}}{z_{1} z_{2}}\right)^{-1} \delta\left(\frac{z_{1}^{-1}-z_{2}^{-1}}{-\frac{z_{0}}{z_{1} z_{2}}}\right) Y_{W}\left(u, z_{1}^{-1}\right) Y_{W}\left(v, z_{2}^{-1}\right) \\
= & \left(z_{1} z_{2}\right)^{-1} z_{2} \delta\left(\frac{z_{2}^{-1}-\frac{z_{0}}{z_{1} z_{2}}}{z_{1}^{-1}}\right) Y_{W}\left(Y\left(u,-\frac{z_{0}}{z_{1} z_{2}}\right) v, z_{2}^{-1}\right) \\
= & z_{1}^{-1} \delta\left(\frac{z_{2}+z_{0}}{z_{1}}\right) Y_{W}\left(Y\left(u,-\frac{z_{0}}{z_{1} z_{2}}\right) v, z_{2}^{-1}\right) .
\end{aligned}
$$

Now, take

$$
u=e^{z_{1} L(1)} e^{\pi i L(0)} z_{1}^{-2 L(0)} a, \quad v=e^{z_{2} L(1)} e^{\pi i L(0)} z_{2}^{-2 L(0)} b .
$$

For proving the desired twisted Jacobi identity, it suffices to prove

$$
\begin{aligned}
& z_{2}^{-1} \delta\left(\frac{z_{1}-z_{0}}{z_{2}}\right)\left(\frac{z_{1}-z_{0}}{z_{2}}\right)^{-2 \alpha} e^{z_{2} L(1)} e^{\pi i L(0)} z_{2}^{-2 L(0)} Y\left(a, z_{0}\right) \\
= & z_{1}^{-1} \delta\left(\frac{z_{2}+z_{0}}{z_{1}}\right) Y\left(e^{z_{1} L(1)} e^{\pi i L(0)} z_{1}^{-2 L(0)} a,-\frac{z_{0}}{z_{1} z_{2}}\right) e^{z_{2} L(1)} e^{\pi i L(0)} z_{2}^{-2 L(0)},
\end{aligned}
$$

or, equivalently to prove

$$
\begin{aligned}
& z_{2}^{-1} \delta\left(\frac{z_{1}-z_{0}}{z_{2}}\right)\left(\frac{z_{1}-z_{0}}{z_{2}}\right)^{-2 \alpha} \cdot \\
& \cdot e^{z_{2} L(1)} e^{\pi i L(0)} z_{2}^{-2 L(0)} Y\left(a, z_{0}\right) z_{2}^{2 L(0)} e^{-\pi i L(0)} e^{-z_{2} L(1)} \\
= & z_{1}^{-1} \delta\left(\frac{z_{2}+z_{0}}{z_{1}}\right) Y\left(e^{z_{1} L(1)} e^{\pi i L(0)} z_{1}^{-2 L(0)} a,-\frac{z_{0}}{z_{1} z_{2}}\right) .
\end{aligned}
$$


Using formulas (2.9) $-(2.12)$, we get

$$
\begin{aligned}
& e^{z_{2} L(1)} e^{\pi i L(0)} z_{2}^{-2 L(0)} Y\left(a, z_{0}\right) z_{2}^{2 L(0)} e^{-\pi i L(0)} e^{-z_{2} L(1)} \\
= & e^{z_{2} L(1)} e^{\pi i L(0)} Y\left(z_{2}^{-2 L(0)} a,-z_{2}^{-2} z_{0}\right) e^{-\pi i L(0)} e^{-z_{2} L(1)} \\
= & e^{z_{2} L(1)} Y\left(e^{\pi i L(0)} z_{2}^{-2 L(0)} a,-z_{2}^{-2} z_{0}\right) e^{-z_{2} L(1)} \\
= & Y\left(e^{z_{2}\left(1+z_{0} z_{2}^{-1}\right) L(1)}\left(1+z_{0} z_{2}^{-1}\right)^{-2 L(0)} e^{\pi i L(0)} z_{2}^{-2 L(0)} a,-\frac{z_{2}^{-2} z_{0}}{1+z_{0} z_{2}^{-1}}\right) \\
= & Y\left(e^{\left(z_{2}+z_{0}\right) L(1)} e^{\pi i L(0)}\left(z_{2}+z_{0}\right)^{-2 L(0)} a,-\frac{z_{0}}{z_{2}\left(z_{2}+z_{0}\right)}\right) .
\end{aligned}
$$

Thus

$$
\begin{aligned}
& z_{2}^{-1} \delta\left(\frac{z_{1}-z_{0}}{z_{2}}\right)\left(\frac{z_{1}-z_{0}}{z_{2}}\right)^{-2 \alpha} \cdot \\
& \cdot e^{z_{2} L(1)} e^{\pi i L(0)} z_{2}^{-2 L(0)} Y\left(a, z_{0}\right) z_{2}^{2 L(0)} e^{-\pi i L(0)} e^{-z_{2} L(1)} \\
= & z_{1}^{-1} \delta\left(\frac{z_{2}+z_{0}}{z_{1}}\right)\left(\frac{z_{2}+z_{0}}{z_{1}}\right)^{2 \alpha} \cdot \\
& \cdot Y\left(e^{\left(z_{2}+z_{0}\right) L(1)} e^{\pi i L(0)}\left(z_{2}+z_{0}\right)^{-2 L(0)} a,-\frac{z_{0}}{z_{2}\left(z_{2}+z_{0}\right)}\right) \\
= & z_{1}^{-1} \delta\left(\frac{z_{2}+z_{0}}{z_{1}}\right) Y\left(e^{z_{1} L(1)} e^{\pi i L(0)} z_{1}^{-2 L(0)} a,-\frac{z_{0}}{z_{2} z_{1}}\right) .
\end{aligned}
$$

Now the proof is complete.

Remark 2.5. Let $V$ be a vertex operator algebra and let $W$ be a $V$-module. It was proved in [FHL] that the double contragredient module $\left(W^{\prime}\right)^{\prime}$ is isomorphic to $W$. Now, assume that $V$ is a $\mathbb{Q}$-graded vertex operator algebra. Following the argument in [FHL], one obtains

$$
\left(Y^{\prime}\right)^{\prime}(v, z)=Y\left(e^{2 \pi i L(0)} v, z\right) \quad(=Y(\tau(v), z)) \quad \text { for } v \in V .
$$

That is, the double contragredient module $\left(W^{\prime}\right)^{\prime}$ is isomorphic to the $\tau$-twist of $W$, which is defined by $W^{\tau}=W$ as a vector space and $Y_{W^{\tau}}(v, z)=Y_{W}(\tau(v), z)$ for $v \in V$.

The following is the main result of this section, which gives a connection between the two constructions of $\sigma_{h}$-twisted modules:

Theorem 2.6. Let $(V, Y, \mathbf{1}, \omega)$ be a vertex operator algebra and let $h \in V$, satisfying (2.2). Set $\tilde{\omega}=\omega+\frac{1}{2} L(-1) h$. Let $W$ be a $V$-module and let $\left(W^{\prime}, Y^{\prime}\right)$ denote the contragredient module of $W$. Identity $W$ with $\left(W^{\prime}\right)^{\prime}$ canonically as a vector space. Then $\left(W,\left(Y^{\prime}\right)_{\tilde{\omega}}^{\prime}\right)$ is isomorphic to $\left(W, Y\left(\Delta(h, z) e^{-\frac{1}{2} \pi i h(0)} \cdot, z\right)\right)$ as a $\sigma_{h}$-twisted $V$-module, where $\left(Y^{\prime}\right)_{\tilde{\omega}}^{\prime}$ denotes the contragredient dual of $Y^{\prime}$ with respect to the conformal vector $\tilde{\omega}$. 
Proof. First from [FHL] we have

$$
z^{L(0)} e^{z_{0} L(1)}=e^{z_{0} z^{-1} L(1)} z^{L(0)} .
$$

With $\tilde{\omega}=\omega+\frac{1}{2} L(-1) h$, we have

$$
\tilde{L}(m)=L(m)-\frac{1}{2}(m+1) h(m) \quad \text { for } m \in \mathbb{Z},
$$

where $Y(\tilde{\omega}, z)=\sum_{n \in \mathbb{Z}} \tilde{L}(n) z^{-n-2}$. In particular, we have

$$
\tilde{L}(-1)=L(-1), \quad \tilde{L}(0)=L(0)-\frac{1}{2} h(0), \quad \tilde{L}(1)=L(1)-h(1) .
$$

Let $a \in V, u \in W, v^{\prime} \in W^{\prime}$. Using (2.7) we have

$$
\begin{aligned}
& \left\langle v^{\prime}, Y(a, z) u\right\rangle \\
= & \left\langle Y^{\prime}\left(e^{z L(1)}\left(-z^{-2}\right)^{L(0)} a, z^{-1}\right) v^{\prime}, u\right\rangle \\
= & \left\langle v^{\prime},\left(Y^{\prime}\right)_{\tilde{\omega}}^{\prime}\left(e^{z^{-1} \tilde{L}(1)} e^{-\pi i \tilde{L}(0)} z^{2 \tilde{L}(0)} e^{z L(1)}\left(-z^{-2}\right)^{L(0)} a, z\right) u\right\rangle .
\end{aligned}
$$

Recall that $[h(0), h(1)]=0$ and $[h(0), L(j)]=0$ for $j=0,1$ and also recall from [FHL] (formula (5.3.1)) that

$$
\left(-z^{2}\right)^{L(0)} e^{z L(1)}\left(-z^{-2}\right)^{L(0)}=e^{-z^{-1} L(1)} .
$$

Then

$$
\begin{aligned}
& e^{z^{-1} \tilde{L}(1)} e^{-\pi i \tilde{L}(0)} z^{2 \tilde{L}(0)} e^{z L(1)}\left(-z^{-2}\right)^{L(0)} \\
= & e^{z^{-1}(L(1)-h(1))} e^{-\pi i\left(L(0)-\frac{1}{2} h(0)\right)} z^{2\left(L(0)-\frac{1}{2} h(0)\right)} e^{z L(1)}\left(-z^{-2}\right)^{L(0)} \\
= & z^{-h(0)} e^{z^{-1}(L(1)-h(1))}\left(-z^{2}\right)^{L(0)} e^{z L(1)}\left(-z^{-2}\right)^{L(0)} e^{\frac{1}{2} \pi i h(0)} \\
= & z^{-h(0)} e^{z^{-1}(L(1)-h(1))} e^{-z^{-1} L(1)} e^{\frac{1}{2} \pi i h(0)} .
\end{aligned}
$$

By Lemma 3.2 from [Li3] we have

$$
e^{z(L(1)-h(1))} e^{-z L(1)}=\exp \left(\sum_{k=1}^{\infty} \frac{h(k)}{k}(-z)^{k}\right) .
$$

Using this we obtain

$$
\begin{aligned}
& \left\langle v^{\prime}, Y(a, z) u\right\rangle \\
= & \left\langle v^{\prime},\left(Y^{\prime}\right)_{\tilde{\omega}}^{\prime}\left(z^{-h(0)} e^{z^{-1}(L(1)-h(1))} e^{-z^{-1} L(1)} e^{\frac{1}{2} \pi i h(0)} a, z\right) u\right\rangle \\
= & \left\langle v^{\prime},\left(Y^{\prime}\right)_{\tilde{\omega}}^{\prime}\left(z^{-h(0)} \exp \left(\sum_{k=1}^{\infty} \frac{h(k)}{k}\left(-z^{-1}\right)^{k}\right) e^{\frac{1}{2} \pi i h(0)} a, z\right) u\right\rangle \\
= & \left\langle v^{\prime},\left(Y^{\prime}\right)_{\tilde{\omega}}^{\prime}\left(\Delta(-h, z) e^{\frac{1}{2} \pi i h(0)} a, z\right) u\right\rangle .
\end{aligned}
$$

Thus we get

$$
\left\langle v^{\prime}, Y\left(\Delta(h, z) e^{-\frac{1}{2} \pi i h(0)} a, z\right) u\right\rangle=\left\langle v^{\prime},\left(Y^{\prime}\right)_{\tilde{\omega}}^{\prime}(a, z) u\right\rangle,
$$

as desired. 


\section{Pseudo-derivations and pseudo-endomorphisms}

In this section, we study what we call $R$-valued pseudo-derivations and pseudoendomorphisms with $R$ a commutative associative algebra over $\mathbb{C}((z))$. These notions naturally extend those of $(\mathbb{C}((z))$-valued $)$ pseudo-derivation in [EK] and pseudoendomorphism in [Li4]. We also study pseudo-endomorphism twists of ordinary representations for a vertex algebra.

Let $R$ be a commutative associative algebra over $\mathbb{C}((z))$, equipped with a $\mathbb{C}$-linear derivation $\partial$ on $R$, satisfying

$$
\partial f(z)=\frac{d}{d z} f(z) \quad \text { for } f(z) \in \mathbb{C}((z)) .
$$

Typical examples of such an $R$ are $\mathbb{C}((z)), \mathbb{C}\left(\left(z^{1 / r}\right)\right)$ with $r$ a positive integer, and $\mathbb{C}\left(\left(z^{1 / r}\right)\right)[\log z]$ with $\log z$ as a new variable where $\partial \log z=z^{-1}$.

We now fix such an $R$ together with $\partial$ throughout this section.

Definition 3.1. Let $V$ be a vertex algebra. An $R$-valued pseudo-derivation of $V$ is an element

$$
\Psi \in \operatorname{Hom}(V, V \otimes R)
$$

satisfying

$$
\Psi Y(u, x) v-(Y(u, x) \otimes 1) \Psi(v)=Y\left(e^{x(1 \otimes \partial)} \Psi(u), x\right)(v \otimes 1)
$$

for $u, v \in V$, where for the expression on right it is understood that

$$
Y(u \otimes a, x)(v \otimes b)=Y(u, x) v \otimes a b
$$

for $u, v \in V, a, b \in R$.

Definition 3.2. Let $V$ be a vertex algebra. An R-valued pseudo-endomorphism of $V$ is an element $\Delta \in \operatorname{Hom}(V, V \otimes R)$, satisfying

$$
\Delta(\mathbf{1})=\mathbf{1} \otimes 1, \quad \Delta Y(v, x)=Y\left(e^{x(1 \otimes \partial)} \Delta(v), x\right) \Delta
$$

for $v \in V$.

As consequences of the definitions we have (cf. [Li4]):

Lemma 3.3. Let $V$ be a vertex algebra. For any $R$-valued pseudo-derivation $\Psi$ of $V$, we have

$$
\Psi(\mathbf{1})=0, \quad(\mathcal{D} \otimes 1) \Psi-\Psi \mathcal{D}=-(1 \otimes \partial) \Psi,
$$

where $\mathcal{D}$ is the linear operator on $V$ defined by $\mathcal{D} v=v_{-2} \mathbf{1}$ for $v \in V$. 
Proof. It is basically the same proof as in [Li4] (Proposition 2.3). We have $\Psi(\mathbf{1})=0$ because

$$
Y\left(e^{x(1 \otimes \partial)} \Psi(\mathbf{1}), x\right)=\Psi Y(\mathbf{1}, x)-(Y(\mathbf{1}, x) \otimes 1) \Psi=\Psi-\Psi=0
$$

and because $Y$ is an injective map. By definition we have

$$
\Psi Y(v, x) \mathbf{1}-(Y(v, x) \otimes 1) \Psi(\mathbf{1})=Y\left(e^{x(1 \otimes \partial)} \Psi(v), x\right)(\mathbf{1} \otimes 1) .
$$

As $\Psi(\mathbf{1})=0$, we get

$$
\Psi Y(v, x) \mathbf{1}=Y\left(e^{x(1 \otimes \partial)} \Psi(v), x\right)(\mathbf{1} \otimes 1)
$$

which implies

$$
\Psi \mathcal{D}(v)=(\mathcal{D} \otimes 1) \Psi(v)+(1 \otimes \partial) \Psi(v)
$$

as we need.

From the argument in the second part we also get:

Lemma 3.4. Let $\Delta$ be an R-valued pseudo-endomorphism of $V$. Then

$$
(\mathcal{D} \otimes 1) \Delta-\Delta \mathcal{D}=-(1 \otimes \partial) \Delta
$$

As $R$ is a commutative associative algebra over $\mathbb{C}$ and $\partial$ is a derivation, by a result of Borcherds [B], $R$ has a vertex algebra structure (over $\mathbb{C}$ ) with 1 as the vacuum vector and with

$$
Y(a, x) b=\left(e^{x \partial} a\right) b=\sum_{n \geq 0} \frac{x^{n}}{n !}\left(\partial^{n} a\right) b
$$

for $a, b \in R$. For convenience, we denote this vertex algebra by $(R, \partial)$. Then for any vertex algebra $V$, we have a tensor product vertex algebra $V \otimes(R, \partial)$, where the vertex operator map, denoted by $Y_{t e n}$, is given by

$$
Y_{\text {ten }}(u \otimes a, x)(v \otimes b)=Y(u, x) v \otimes Y(a, x) b=Y(u, x) v \otimes\left(e^{x \partial} a\right) b
$$

for $u, v \in V, a, b \in R$. Identify $V$ as a vertex subalgebra of $V \otimes(R, \partial)$ in the canonical way, so that $V \otimes(R, \partial)$ is a natural $V$-module. A linear map $\Phi: V \rightarrow V \otimes R$ is called a derivation if

$$
\Phi Y(u, x) v=Y(u, x) \Psi(v)+Y_{\text {ten }}(\Phi(u), x) v
$$

for $u, v \in V$.

Now, we interpret $R$-valued pseudo-derivations and -endomorphisms as ordinary derivations and homomorphisms of vertex algebras. 
Lemma 3.5. Let $V$ be a vertex algebra. An R-valued pseudo-derivation (resp. pseudo-endomorphism) of $V$ exactly amounts to a derivation (resp. an endomorphism) from $V$ to the tensor product vertex algebra $V \otimes(R, \partial)$.

Proof. Let $\Psi: V \rightarrow V \otimes R$ be a linear map. Notice that

$$
Y\left(e^{x(1 \otimes \partial)}(w \otimes a), x\right)=Y(w, x) \otimes Y(a, x)=Y_{\text {ten }}(w \otimes a, x)
$$

for $w \in V, a \in R$. Then (3.1) exactly amounts to

$$
\Psi Y(v, x) u-(Y(v, x) \otimes 1) \Psi(u)=Y_{\text {ten }}(\Psi(v), x)(u \otimes 1) .
$$

Thus $\Psi$ is an $R$-valued pseudo-derivation if and only if $\Psi$ is a derivation of $V$ modules. The assertion for pseudo-endomorphism is also clear.

Using Lemma 3.5 we have (cf. [Li4]):

Proposition 3.6. Let $V$ be a vertex algebra. The restriction of every derivation of the tensor product vertex algebra $V \otimes(R, \partial)$ is an $R$-valued pseudo-derivation (resp. pseudo-endomorphism) of $V$. Conversely, the unique $R$-linear extension of any pseudo-derivation (resp. pseudo-endomorphism) of $V$ is a derivation (resp. endomorphism) of $V \otimes(R, \partial)$.

Definition 3.7. A pseudo-endomorphism $\Delta$ of a vertex algebra $V$ is called a pseudoautomorphism if the $R$-linear extension of $\Delta$ is an automorphism of the tensor product vertex algebra $V \otimes(R, \partial)$.

Remark 3.8. Consider the case $R=\mathbb{C}((z))$ with $\partial=\frac{d}{d z}$. We denote a pseudoderivation by $\Psi(z)$ to show its dependence of variable $z$. As

$$
e^{x \frac{d}{d z}} \Psi(z)=\Psi(z+x),
$$

we have

$$
\Psi(z) Y(v, x)-(Y(v, x) \otimes 1) \Psi(z)=Y(\Psi(z+x) v, x)
$$

for $v \in V$. With Lemma 3.3, we see that a $\mathbb{C}((z))$-valued pseudo-derivation is exactly a pseudo-derivation in the sense of [EK]. Similarly, a $\mathbb{C}((z))$-valued pseudoendomorphism is exactly a pseudo-endomorphism in the sense of [Li4].

Remark 3.9. Let $V$ be a vertex operator algebra and let $h \in V_{(1)}$ be such that

$$
L(n) h=\delta_{n, 0} h, \quad h_{n} h=\delta_{n, 1} \gamma \mathbf{1}
$$

for $n \geq 0$, where $\gamma$ is a rational number. Assume that $h_{0}$ acts on $V$ semisimply with eigenvalues in $\frac{1}{r} \mathbb{Z}$ for some positive integer $r$. Recall

$$
\Delta(h, z)=z^{h(0)} \exp \left(\sum_{k=1}^{\infty} \frac{h(k)}{-k}(-z)^{-k}\right) \in(\operatorname{End} V)\left[\left[z^{1 / r}, z^{-1 / r}\right]\right] .
$$

The proof of Proposition 5.4 of [Li1] is mainly to prove that $\Delta(h, z)$ is a $\mathbb{C}\left(\left(z^{1 / r}\right)\right)$ valued pseudo-endomorphism. 
Just as with the classical case, the exponentials of pseudo-derivations should give rise to pseudo-automorphisms.

Lemma 3.10. Let $V$ be a vertex algebra and let $\Phi$ be an $R$-valued pseudo-derivation of $V$, satisfying the condition that for every $v \in V$, there exists a finite-dimensional subspace $U$ of $V$, containing $v$, such that

$$
\Phi^{n+k}(U) \subset U \otimes I^{n} \quad \text { for } n \geq 0
$$

where $k$ is a fixed nonnegative integer and $I$ is a fixed ideal of $R$. Suppose that $R$ is I-adically complete. Then

$$
\exp \Phi=\sum_{n \geq 0} \frac{1}{n !} \Phi^{n}
$$

is an $R$-valued pseudo-automorphism of $V$.

Proof. Notice that the assumptions imply that $\sum_{n \geq 0} \frac{1}{n !} \Phi^{n}(v)$ lies in $V \otimes R$ for $v \in V$, so that $\exp \Phi$ is an $R$-linear automorphism of $V \otimes R$. By Proposition 3.6, $\Phi$, after $R$-linearly extended, is a derivation of the vertex algebra $V \otimes(R, \partial)$. Since $\Phi \mathbf{1}=0$ (by Lemma 3.3), we have $(\exp \Phi) \mathbf{1}=\mathbf{1}$. Let $m \in \mathbb{Z}$. Just as with any non-associative algebra, we have

$$
(\exp \Phi)\left(a_{m} b\right)=(\exp \Phi)(a)_{m}(\exp \Phi)(b) \quad \text { for } a, b \in V \otimes R
$$

This shows that $\exp \Phi$ is an automorphism of vertex algebra $V \otimes(R, \partial)$. By Proposition 3.6, $\exp \Phi$ is an $R$-valued pseudo-automorphism of $V$.

The following result, which slightly generalizes Proposition 1.9 of [EK] with a different proof, enables us to construct a family of pseudo-derivations:

Proposition 3.11. Let $V$ be a vertex algebra. For any $v \in V, f \in R$, set

$$
X_{v, f}=\sum_{n \geq 0} \frac{1}{n !}\left(\partial^{n} f\right) v_{n}
$$

Then $X_{v, f}$ is an $R$-valued pseudo-derivation of $V$.

Proof. Note that $(v \otimes f)_{0}$, which is the coefficient of $x^{-1}$ in the vertex operator $Y_{\text {ten }}(v \otimes f, x)$, is a derivation of $V \otimes(R, \partial)$. In view of Proposition 3.6, $(v \otimes f)_{0}$ is an $R$-valued pseudo-derivation of $V$. On the other hand, we have

$$
(v \otimes f)_{0}=\operatorname{Res}_{x} Y_{\text {ten }}(v \otimes f, x)=\operatorname{Res}_{x} Y(v, x) \otimes e^{x \partial} f=X_{v, f} .
$$

Now the assertion follows.

In view of Proposition 3.11 and Lemma 3.10 we immediately have: 
Corollary 3.12. Let $V$ be a vertex algebra and let $v \in V$. Suppose that for any $w \in V$, there exists a positive integer $k$ such that

$$
v_{n_{1}} v_{n_{2}} \cdots v_{n_{r}} w=0
$$

for any nonnegative integers $n_{1}, \ldots, n_{r}$ with $n_{1}+\cdots+n_{r} \geq k$. Then for any $f \in R$,

$$
\exp \left(\sum_{n \geq 0} \frac{1}{n !}\left(\partial^{n} f\right) v_{n}\right)
$$

is an R-valued pseudo-automorphism of $V$. In particular, this is true if $V$ is a vertex operator algebra and if $v$ is homogeneous with a non-positive conformal weight.

Proof. The first assertion is immediate. For the second assertion, as wt $v \leq 0$, we have wt $v_{n}=$ wt $v-n-1<0$ for $n \geq 0$. Since $V$ is truncated from below by definition, the assumption in the first part is satisfied. Then it follows.

Proposition 3.13. Let $V$ be a vertex operator algebra and let $v \in V$ be homogeneous of weight $k$ positive, such that $\left[v_{m}, v_{n}\right]=0$ for $m, n \geq 0$. Suppose that $v_{0}, v_{1}, \ldots, v_{k-1}$ are locally finite on $V$ and suppose that $f(z) \in z^{k} \mathbb{C}[[z]]$. Then $\exp X_{v, f}$ exists in $\operatorname{Hom}(V, V \otimes \mathbb{C}((z)))$ and it is a $\mathbb{C}((z))$-valued pseudo-automorphism of $V$.

Proof. As $f^{(n)}(z) \in z \mathbb{C}[[z]]$ for $0 \leq n \leq k-1$ and as $v_{0}, v_{1}, \ldots, v_{k-1}$ are locally finite, we see that

$$
\exp \left(\sum_{n=0}^{k-1} \frac{1}{n !} f^{(n)}(z) v_{n}\right)
$$

exists in $\operatorname{Hom}(V, V \otimes \mathbb{C}[[z]])$ (not just in $(\operatorname{End} V)[[z]])$. On the other hand,

$$
\exp \left(\sum_{n \geq k} \frac{1}{n !} f^{(n)}(z) v_{n}\right)
$$

also exists in $\operatorname{Hom}(V, V \otimes \mathbb{C}[[z]])$ as wt $v_{n}=k-n-1<0$ for $n \geq k$. Consequently, $\exp X_{v, f}$ exists in $\operatorname{Hom}(V, V \otimes \mathbb{C}((z)))$ and it is a $\mathbb{C}((z))$-valued pseudo-automorphism.

Example 3.14. Let $V$ be a vertex operator algebra and let $v \in V_{(1)}$ be such that $\left[v_{m}, v_{n}\right]=0$ for $m, n \geq 0$ and such that $v_{0}$ is locally finite on $V$. Let $r$ be a positive integer and let $f(z)=z^{1 / r}$. We have

$$
X_{v, f}=\sum_{n \geq 0}\left(\begin{array}{c}
\frac{1}{r} \\
n
\end{array}\right) v_{n} z^{-n+\frac{1}{r}} .
$$

Note that $\exp \left(v_{0} z^{1 / r}\right)$ exists in $\operatorname{Hom}\left(V, V \otimes \mathbb{C}\left[\left[z^{1 / r}\right]\right]\right)$ and $\exp \left(\sum_{n \geq 1}\left(\begin{array}{c}\frac{1}{r} \\ n\end{array}\right) v_{n} z^{-n+\frac{1}{r}}\right)$ exists in $\operatorname{Hom}\left(V, V \otimes \mathbb{C}\left[z^{-1 / r}\right]\right)$. Then $\exp \left(X_{v, f}\right)$ exists in $\operatorname{Hom}\left(V, V \otimes \mathbb{C}\left(\left(z^{1 / r}\right)\right)\right)$ and it is a $\mathbb{C}\left(\left(z^{1 / r}\right)\right)$-valued pseudo-automorphism of $V$. 
The importance of pseudo-endomorphisms can be seen from the following results due to [Li2] and [DLM1] (cf. [Li4]):

Proposition 3.15. Let $V$ be a vertex algebra and let $\Delta(z)$ be a $(\mathbb{C}((z))$-valued $)$ pseudo-endomorphism of $V$. Then for any $V$-module $\left(W, Y_{W}\right),\left(W, Y_{W}^{\Delta}\right)$ carries the structure of a $V$-module where

$$
Y_{W}^{\Delta}(v, x)=Y_{W}(\Delta(x) v, x) \quad \text { for } v \in V .
$$

Proposition 3.16. Let $V$ be a vertex algebra and let $\sigma$ be an automorphism of $V$ of order $r$. Suppose that $\Delta(z)$ is a $\mathbb{C}\left(\left(z^{1 / r}\right)\right)$-valued pseudo-endomorphism of $V$, satisfying

$$
(\sigma \times \tau) \Delta(z)=\Delta(z),
$$

where $\tau f\left(z^{1 / r}\right)=f\left(\omega_{r} z^{1 / r}\right)$ for $f(z) \in \mathbb{C}((z))$. Then for any $V$-module $\left(W, Y_{W}\right)$, $\left(W, Y_{W}^{\Delta}\right)$ carries the structure of a $\sigma$-twisted $V$-module where

$$
Y_{W}^{\Delta}(v, x)=Y_{W}(\Delta(x) v, x) \quad \text { for } v \in V .
$$

Example 3.17. Let $V=M(1)$ be the (free field) Heisenberg vertex operator algebra with one generator $h$ satisfying

$$
\left[h_{m}, h_{n}\right]=m \delta_{m+n, 0}
$$

for $m, n \in \mathbb{Z}$. Note that $h_{0}=0$ on $M(1)$. We see that the assumption in Corollary 3.12 with $v=h$ is satisfied. Thus for any $f \in \mathbb{C}((z))$, $\exp X_{h, f}$, lying in $\operatorname{Hom}(M(1), M(1) \otimes \mathbb{C}((z)))$, is a pseudo-automorphism of $M(1)$. Let $k$ be a positive integer and set

$$
\Phi_{k}(z)=\exp \left(X_{h, z^{-k}}\right)=\exp \left(\sum_{n \geq 1}\left(\begin{array}{c}
-k \\
n
\end{array}\right) h(n) z^{-n-k}\right) .
$$

Noticing that $X_{h, z^{-k}}(h)=-k z^{-k-1} \mathbf{1}$, we have $\Phi_{k}(z)(h)=h-k z^{-k-1} \mathbf{1}$. For any $M(1)$-module $\left(W, Y_{W}\right)$, we have a module $\left(W, Y_{W}^{\Phi_{k}}\right)$ for $M(1)$ viewed as a vertex algebra, where

$$
Y_{W}^{\Phi_{k}}(h, z)=Y_{W}\left(\Phi_{k}(z) h, z\right)=Y_{W}(h, z)-k z^{-k-1} .
$$

Set $Y_{W}^{\Phi_{k}}(h, z)=\sum_{n \in \mathbb{Z}} h_{n}^{\Phi_{k}} z^{-n-1}$. We have

$$
h_{n}^{\Phi_{k}}=h_{n}-k \delta_{n, k} \quad \text { for } n \in \mathbb{Z} .
$$

Take $W=M(1)$, the adjoint module, on which $h_{k}$ is locally nilpotent. We see that $h_{k}^{\Phi_{k}}=h_{k}-k$ is locally finite but not nilpotent. Thus the deformed $M(1)$-module $\left(M(1), Y^{\Phi_{k}}\right)$ is not a highest weight module. Such $M(1)$-modules viewed as modules for the Heisenberg algebra were studied in [LW]. 
Next, we interpret $\Delta(h, z)$ (recall Remark 3.9) as the exponential of a pseudoderivation. First we define an algebra $R$ involving $\log z$. Let $r$ be a positive integer. Consider algebra $\mathbb{C}\left(\left(z^{1 / r}\right)\right)[[y]]$ with $y$ an independent variable. There exists a $\mathbb{C}$ linear derivation $\partial$ such that

$$
\partial\left(\sum_{n \geq 0} g_{n} y^{n}\right)=\sum_{n \geq 0}\left(\frac{d g_{n}}{d z} y^{n}+n z^{-1} g_{n} y^{n-1}\right)
$$

for $\sum_{n>0} g_{n} y^{n} \in \mathbb{C}\left(\left(z^{1 / r}\right)\right)[[y]]$. In particular, we have $\partial y=z^{-1}$. The algebra $\mathbb{C}\left(\left(z^{1 / r}\right)\right)[[y]]$ contains $\mathbb{C}((z)) \otimes \mathbb{C}[[y]], \mathbb{C}\left[z, z^{-1}\right] \otimes \mathbb{C}[[y]]$, and $\mathbb{C}\left[y, z^{-1}\right]$ as subalgebras which are all stable under the derivation operator $\partial$.

Set

$$
K_{r}[y]=\left\langle y, e^{\alpha y} \mid \alpha \in \frac{1}{r} \mathbb{Z}\right\rangle,
$$

a subalgebra of $\mathbb{C}[[y]]$, where

$$
e^{\alpha y}=\sum_{n \geq 0} \frac{1}{n !} \alpha^{n} z^{n} \in \mathbb{C}[[z]] .
$$

One can show that $K_{r}[y]$ is isomorphic to $\mathbb{C}\left[\frac{1}{r} \mathbb{Z}\right] \otimes \mathbb{C}[y]$, where $\mathbb{C}\left[\frac{1}{r} \mathbb{Z}\right]$ stands for the group algebra of $\frac{1}{r} \mathbb{Z}$.

Note that both $\mathbb{C}((z)) \otimes K_{r}[y]$ and $\mathbb{C}\left(\left(z^{1 / r}\right)\right)[y]$ are subalgebras of $\mathbb{C}\left(\left(z^{1 / r}\right)\right)[[y]]$, which are stable under $\partial$. The following is straightforward:

Lemma 3.18. There exists an algebra homomorphism

$$
\theta: \mathbb{C}((z)) \otimes K_{r}[y] \rightarrow \mathbb{C}\left(\left(z^{1 / r}\right)\right)[y]
$$

which is uniquely determined by

$$
\theta(f(z))=f(z), \quad \theta(y)=y, \quad \theta\left(e^{ \pm y / r}\right)=z^{ \pm 1 / r}
$$

for $f(z) \in \mathbb{C}((z))$. Furthermore, we have $\theta \partial=\partial \theta$.

For any vertex algebra $V$ and for any $v \in V$, by Proposition 3.11 ,

$$
X_{v, y}=\sum_{n \geq 0} \frac{1}{n !}\left(\partial^{n} y\right) v_{n}=v_{0} y+\sum_{n \geq 1}(-1)^{n-1} \frac{1}{n} v_{n} z^{-n}
$$

is a $\mathbb{C}\left[y, z^{-1}\right]$-valued pseudo-derivation of $V$.

Proposition 3.19. Let $V$ be a vertex operator algebra and let $h \in V_{(1)}$, satisfying that $\left[h_{m}, h_{n}\right]=0$ for $m, n \geq 0$ and that $h_{0}$ is locally finite on $V$. Then $\exp \left(X_{h, y}\right)$ is $a \mathbb{C}\left[z^{-1}\right] \otimes \mathbb{C}[[y]]$-valued pseudo-automorphism of $V$. Furthermore, if the eigenvalues of $h_{0}$ lie in $\frac{1}{r} \mathbb{Z}, \exp \left(X_{h, z}\right)$ is a $\mathbb{C}\left[z^{-1}\right] \otimes K_{r}[y]$-valued pseudo-endomorphism of $V$. 
Proof. Since wt $h_{n}=-n \leq 1$ for $n \geq 1$, we see that $\sum_{n>1}(-1)^{n-1} \frac{1}{n} h_{n} z^{-n}$ is locally nilpotent on $V$. As $h_{0}$ is assumed to be locally finite on $V$, we have

$$
e^{y h_{0}} \in \operatorname{Hom}(V, V \otimes \mathbb{C}[[y]])
$$

Thus

$$
\exp \left(X_{h, y}\right) \in \operatorname{Hom}\left(V, V \otimes \mathbb{C}\left[z^{-1}\right] \otimes \mathbb{C}[[y]]\right) .
$$

We see that $\exp \left(X_{h, y}\right)$ is a $\mathbb{C}\left[z^{-1}\right] \otimes \mathbb{C}[[y]]$-valued pseudo-endomorphism of $V$.

Now, assume that the eigenvalues of $h_{0}$ lie in $\frac{1}{r} \mathbb{Z}$. By using the semisimplenilpotent decomposition of $h_{0}$, we have

$$
e^{y h_{0}} \in \operatorname{Hom}\left(V, V \otimes K_{r}[y]\right) .
$$

Consequently, we obtain

$$
\exp \left(X_{h, y}\right) \in \operatorname{Hom}\left(V, V \otimes \mathbb{C}\left[z^{-1}\right] \otimes K_{r}[y]\right)
$$

proving that $\exp \left(X_{h, z}\right)$ is a $\mathbb{C}\left[z^{-1}\right] \otimes K_{r}[y]$-valued pseudo-endomorphism of $V$.

Identify $\mathbb{C}\left(\left(z^{1 / r}\right)\right)[\log z]$ with the subalgebra $\mathbb{C}\left(\left(z^{1 / r}\right)\right)[y]$ of $\mathbb{C}\left(\left(z^{1 / r}\right)\right)[[y]]$ where $y=\log z$. That is, $\log z$ is considered as a formal variable. We have (cf. [AM], [H]):

Corollary 3.20. Let $V$ be a vertex operator algebra and let $h \in V_{(1)}$, satisfying that $\left[h_{m}, h_{n}\right]=0$ for $m, n \geq 0$ and that $h_{0}$ is locally finite on $V$ with eigenvalues lying in $\frac{1}{r} \mathbb{Z}$. Set

$$
\Delta(h, z)=\exp \left(h_{0} \log z\right) \exp \left(\sum_{n \geq 1}(-1)^{n-1} \frac{1}{n} h_{n} z^{-n}\right),
$$

where for $v \in V, e^{h_{0} \log z} v$ is defined by

$$
e^{h_{0} \log z} v=z^{h_{0}^{s s}} e^{h_{0}^{n}} v
$$

where $h_{0}=h_{0}^{s s}+h_{0}^{n}$ is the semisimple-nilpotent decomposition on any finite-dimensional $h_{0}$-stable subspace $U$ containing $v$. Then $\Delta(h, z)$ is a $\mathbb{C}\left(\left(z^{1 / r}\right)\right)[\log z]$-valued pseudoendomorphism of $V$.

Proof. It is clear that the definition does not depend on the choice of $U$. By Proposition [3.19, $\exp \left(X_{h, y}\right)$ is a $\mathbb{C}\left[z^{-1}\right] \otimes K_{r}[y]$-valued pseudo-endomorphism of $V$. In view of Lemma [3.5, $\exp \left(X_{h, y}\right)$ is a vertex algebra homomorphism from $V$ to $V \otimes\left(\mathbb{C}\left[z^{-1}\right] \otimes K_{r}[y], \partial\right)$. On the other hand, from Lemma 3.18, we see that the algebra homomorphism $\theta$ is actually a vertex algebra homomorphism from $\left(\mathbb{C}((z)) \otimes K_{r}[y], \partial\right)$ to $\left(\mathbb{C}\left(\left(z^{1 / r}\right)\right)[y], \partial\right)$. Then $\theta \circ \exp \left(X_{h, y}\right)$ is a vertex algebra homomorphism from $V$ to $V \otimes\left(\mathbb{C}\left(\left(z^{1 / r}\right)\right)[y], \partial\right)$ and by Lemma 3.5 it is a $\mathbb{C}\left(\left(z^{1 / r}\right)\right)[y]$-valued pseudoendomorphism of $V$. 
Now we consider pseudo-endomorphism twists of representations for a vertex algebra. Recall from [LTW] (Remark 2.16) the following result on the definition of a twisted module (cf. [Li1]):

Proposition 3.21. Let $V$ be a vertex algebra and let $\sigma$ be an automorphism of $V$ of order $r$. Set

$$
\omega_{r}=\exp (2 \pi \sqrt{-1} / r) .
$$

Let $W$ be a vector space and let

$$
Y_{W}: V \rightarrow \operatorname{Hom}\left(W, W\left(\left(x^{\frac{1}{r}}\right)\right)\right) \subset(\operatorname{End} W)\left[\left[x^{\frac{1}{r}}, x^{-\frac{1}{r}}\right]\right]
$$

be a linear map. Then $\left(W, Y_{W}\right)$ carries the structure of a $\sigma$-twisted $V$-module if and only if the following conditions hold: $Y_{W}(\mathbf{1}, x)=1_{W}$,

$$
Y_{W}(\sigma v, x)=\lim _{x^{\frac{1}{r}} \rightarrow \omega_{r}^{-1} x^{\frac{1}{r}}} Y_{W}(v, x)
$$

for $v \in V$, and for $u, v \in V$, there exists $k \in \mathbb{N}$ such that

$$
\left(x_{1}-x_{2}\right)^{k} Y_{W}\left(u, x_{1}\right) Y_{W}\left(v, x_{2}\right) \in \operatorname{Hom}\left(W, W\left(\left(x_{1}^{\frac{1}{r}}, x_{2}^{\frac{1}{r}}\right)\right)\right)
$$

and

$$
x_{0}^{k} Y_{W}\left(Y\left(u, x_{0}\right) v, x_{2}\right)=\left.\left(\left(x_{1}-x_{2}\right)^{k} Y_{W}\left(u, x_{1}\right) Y_{W}\left(v, x_{2}\right)\right)\right|_{x_{1}^{1 / r}=\left(x_{2}+x_{0}\right)^{1 / r}} .
$$

Definition 3.22. Let $V$ be a vertex algebra and let $r$ be a positive integer. A $\mathbb{C}\left(\left(z^{1 / r}\right)\right)$-valued $V$-module is a vector space $W$ equipped with a linear map

$$
Y_{W}: V \rightarrow \operatorname{Hom}\left(W, W\left(\left(x^{1 / r}\right)\right)\right) \subset(\operatorname{End} W)\left[\left[x^{\frac{1}{r}}, x^{-\frac{1}{r}}\right]\right]
$$

satisfying all the conditions listed in Proposition 3.21 except (3.12).

The following generalizes Proposition 3.16 in a certain way (cf. $[\mathrm{H}]$ ):

Proposition 3.23. Let $V$ be a vertex algebra and let $\Delta(z)$ be a $\mathbb{C}\left(\left(z^{1 / r}\right)\right)$-valued pseudo-endomorphism of $V$ with $r$ a positive integer. Let $\left(W, Y_{W}\right)$ be a $V$-module. For $v \in V$, define

$$
Y_{W}^{\Delta}(v, x)=Y_{W}(\Delta(x) v, x)
$$

Then $\left(W, Y_{W}^{\Delta}\right)$ carries the structure of a $\mathbb{C}\left(\left(z^{1 / r}\right)\right)$-valued $V$-module.

Proof. For $v \in V, w \in W$, as $\Delta(x) v \in V \otimes \mathbb{C}\left(\left(x^{1 / r}\right)\right)$, we have $Y_{W}^{\Delta}(v, x) w \in$ $W\left(\left(x^{1 / r}\right)\right)$. We have

$$
Y_{W}^{\Delta}(\mathbf{1}, x)=Y_{W}(\Delta(x)(\mathbf{1}), x)=Y_{W}(\mathbf{1}, x)=1_{W}
$$


Let $u, v \in V$. As $\Delta(z)(u), \Delta(z)(v) \in V \otimes \mathbb{C}\left(\left(z^{1 / r}\right)\right)$, there exists a nonnegative integer $k$ such that

$$
\left(x_{1}-x_{2}\right)^{k}\left[Y_{W}\left(\Delta\left(z_{1}\right) u, x_{1}\right), Y_{W}\left(\Delta\left(z_{2}\right) v, x_{2}\right)\right]=0 .
$$

Substituting $z_{1}^{1 / r}=x_{1}^{1 / r}, z_{2}^{1 / r}=x_{2}^{1 / r}$, we get

$$
\left(x_{1}-x_{2}\right)^{k}\left[Y_{W}^{\Delta}\left(u, x_{1}\right), Y_{W}^{\Delta}\left(v, x_{2}\right)\right]=0,
$$

which implies

$$
\left(x_{1}-x_{2}\right)^{k} Y_{W}^{\Delta}\left(u, x_{1}\right) Y_{W}^{\Delta}\left(v, x_{2}\right) \in \operatorname{Hom}\left(W, W\left(\left(x_{1}^{1 / r}, x_{2}^{1 / r}\right)\right)\right) .
$$

We also have

$$
\begin{aligned}
& x_{0}^{k} Y_{W}\left(Y\left(\Delta\left(z_{1}\right) u, x_{0}\right) \Delta\left(z_{2}\right) v, x_{2}\right) \\
= & \left.\left(\left(x_{1}-x_{2}\right)^{k} Y_{W}\left(\Delta\left(z_{1}\right) u, x_{1}\right) Y_{W}\left(\Delta\left(z_{2}\right) v, x_{2}\right)\right)\right|_{x_{1}^{1 / r}=\left(x_{2}+x_{0}\right)^{1 / r}} .
\end{aligned}
$$

Substituting $z_{1}^{1 / r}=\left(x_{2}+x_{0}\right)^{1 / r}, z_{2}^{1 / r}=x_{2}^{1 / r}$, we get

$$
x_{0}^{k} Y_{W}^{\Delta}\left(Y\left(u, x_{0}\right) v, x_{2}\right)=\left.\left(\left(x_{1}-x_{2}\right)^{k} Y_{W}^{\Delta}\left(u, x_{1}\right) Y_{W}^{\Delta}\left(v, x_{2}\right)\right)\right|_{x_{1}^{1 / r}=\left(x_{2}+x_{0}\right)^{1 / r}},
$$

noticing that

$$
\begin{aligned}
& x_{0}^{k} Y_{W}\left(Y\left(\Delta\left(x_{2}+x_{0}\right) u, x_{0}\right) \Delta\left(x_{2}\right) v, x_{2}\right) \\
= & x_{0}^{k} Y_{W}\left(\Delta\left(x_{2}\right) Y\left(u, x_{0}\right) v, x_{2}\right) \\
= & x_{0}^{k} Y_{W}^{\Delta}\left(Y\left(u, x_{0}\right) v, x_{2}\right) .
\end{aligned}
$$

This completes the proof.

Remark 3.24. Let $R$ be a commutative algebra over $\mathbb{C}((z))$ as before. For a vertex algebra $V$, one can define a notion of $R$-valued $V$-module.

\section{References}

[AM] D. Adamovic and A. Milas, Lattice construction of Logarithmic modules for certain vertex algebras, Selecta Math. (New Series) 15 (2009) 535-561.

[B] R. E. Borcherds, Vertex algebras, Kac-Moody algebras, and the Monster, Proc. Natl. Acad. Sci. USA 83 (1986) 3068-3071.

[D] C. Dong, Twisted modules for vertex operator algebras associated with even lattices, J. Algebra 165 (1994) 90-112.

[DLM1] C. Dong, H.-S. Li and G. Mason, Simple currents and extensions of vertex operator algebras, Commun. Math. Phys. 180 (1996) 671-707. 
[DLM2] C. Dong, H.-S. Li and G. Mason, Vertex operator algebras associated to admissible $\hat{s l}_{2}$-modules, Commun. Math. Phys. 184 (1997) 65-93.

[DLM3] C. Dong, H.-S. Li and G. Mason, Twisted representations of vertex operator algebras, Math. Ann. 310 (1998) 571-600.

[DLinM] C. Dong, Z. Lin and G. Mason, On vertex operator algebras as $s l_{2}$-modules, in Proc. on groups and related topics, Columbus, May, 1993, ed. by K. Harada, S. Sehgal and R. Solomon, Walter de Gruyter, Berlin-New York.

[EK] P. Etingof and D. Kazhdan, Quantization of Lie bialgebras, V, Selecta Math. (New Series) 6 (2000) 105-130.

[FFR] Alex J. Feingold, Igor B. Frenkel and John F. X. Ries, Spinor Construction of Vertex Operator Algebras, Triality, and $E_{8}^{(1)}$, Contemporary Math. 121 (1991).

[FRS] K. Fredenhagen, K.-H. Rehren and B. Schroer, Superselection sectors with Braid group statistics and exchange algebras I, Comm. Math. Phys. 125 (1989) 201-226.

[FHL] I. Frenkel, Y.-Z. Huang and J. Lepowsky, On axiomatic approaches to vertex operator algebras and modules, Memoirs Amer. Math. Soc. 104, 1993.

[FLM1] I. B. Frenkel, J. Lepowsky and A. Meurman, A natural representation of the Fischer-Griess Monster with the modular function $J$ as character, Proc. Natl. Acad. Sci. USA 81 (1984) 3256-3260.

[FLM2] I. Frenkel, J. Lepowsky and A. Meurman, Vertex Operator Algebras and the Monster, Pure and Appl. Math. Vol. 134, Academic Press, Boston, 1988 .

[HK] Hagg, R., Kastler, D., An algebraic approach to field theory, J. Math. Phys. 5 (1964) 848-861.

[H] Y.-Z. Huang, Generalized twisted modules associated to general automorphisms of a vertex operator algebra, Commun. Math. Phys. (2010); arXiv:0905.0514.

[Le] J. Lepowsky, Calculus of twisted vertex operators, Proc. Natl. Acad. Sci. USA 82 (1985) 8295-8299.

[Li1] H.-S. Li, Local systems of twisted vertex operators, vertex superalgebras and twisted modules, in: Moonshine, the Monster and Related Topics, Proc. Joint Summer Research Conference, Mount Holyoke, 1994, ed. by C. Dong and G. Mason, Contemporary Math. 193, Amer. Math. Soc., Providence, 1996, 203-236. 
[Li2] H.-S. Li, The physics superselection principle in vertex operator algebra theory, J. Algebra 196 (1997) 436-457.

[Li3] H.-S. Li, Extension of vertex operator algebras by a self-dual simple module, J. Algebra 187 (1997) 236-267.

[Li4] H.-S. Li, Pseudoderivations, pseudoautomorphisms and simple current modules for vertex operator algebras, in Proceedings of the International Conference on "Infinite Dimensional Aspects of Representation Theory and Applications," University of Virginia, Charlottesville, May 18-22, 2004, Contemporary Math 392 (2005), 55-65.

[Li5] H.-S. Li, A smash product construction of nonlocal vertex algebras, Commun. Contemporary Math. 9 (2007) 605-637.

[LTW] H.-S. Li, Shaobin Tan, and Qing Wang, Twisted modules for quantum vertex algebras, J. Pure Applied Algebra 214 (2010) 201-220.

[LW] H.-S. Li and Qing Wang, On vertex algebras and their modules associated with even lattices, J. Pure Applied Algebra 213 (2009) 1097-111. 